$$
\text { C6. } \left.C_{\text {BNL-43084 }}-926\right) / 60-5
$$

\title{
Flectron Yield XAFS Study of Evaporated Co/Pd Multilayers with Various Thichinese :-:stios of Co to Pd Sublayers: Simulations of the Co K-edge XAFS and Fourier Transforms
}

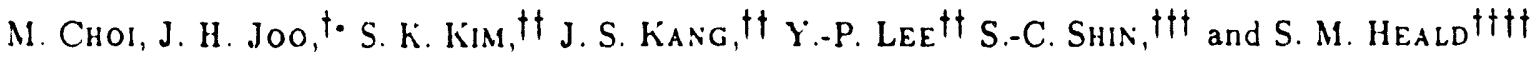 \\ Department of Physics, Catholic Viniversity Medical College, Seosl, 197.701. Horea \\ † Materials Design Laboratory, Korea Institute of Science and Technology, Seoul, 196.791, Korea \\ t† Basic Science Branch, Research Institute of Industrial Science and Technology, Pohang, 790.600, Korea \\ ttt Depertment of Physics, Korea Advanced Institute of Science and Technology, Tacjeon, 905.701, Korea \\ †t† Brookhaven National Láboratory, Upton, NY 11979, USA
}

\begin{abstract}
Electron-yield XAFS measurements were made on e-beam evaporaled $\mathrm{Co} / \mathrm{Pd}$ multilayers with various sublayer thicknesses and different thickness ratios of Co to Pd sublayers. The Co K-edge and the Pd K-edge XAFS dats were obtained for the $C o / P d$ multilayers with the sublayer thicknesses of $3 \AA / 4 \AA, 15 \AA / 4 \AA, 3 \AA / 15 \AA, 2.1 A / 13.5 \AA$, and $2.2 \AA / 4.5 \AA$. The Fourier transforms of the Co K XAFS for most samples show a splitting of major peak, and the magnitude ratio of these split peaks varies systematically with the thickness ratio of the Pd sublayer to the Co sublayer, whereas the Fourier transforms of the Pd K XAFS for the same samples do not show a splitting of peaks. As a preliminary analysis, the Co K XAFS and the split peaks in the Fourier transform for the $\mathrm{Co} / \mathrm{Pd}(3 \AA / 4 \AA)$ case were simulated by using the FEFF calculations, and the Co $K X A F S$ and the major peak in the fourier transform for the $\mathrm{Co} / \mathrm{Pd}(15 \AA / 4 \AA)$ case were also simulated consiztently.
\end{abstract}

KEYWORDS: Electron-yield XAFS, multilayers, FEFF

\section{\$1. Introduction}

$\mathrm{Co} / \mathrm{Pd}$ multilayers with a perpendicular magnetic anisotropy are considered to be promising as magnetooptical recording device materials. The magnetic and magnetooptical properties of $\mathrm{Co} / \mathrm{Pd}$ multilayers have recently been studied extensively, ${ }^{1-3)}$ and the properties such as the magnetic anisotropy, the coercivity, and the Kerr rotation which are useful for magnetooptical recording have been shown to depend strongly on the sublayer thickness and on the interdiffusion at the interfaces. The tunability of these properties is valuable in practical applications, but the microstructural aspect of this sublayer thickness dependence has not been studied fully. Especially, the perpendicular anisotropy is deemed to originate from the interface anisotropy. However, the atomic structure and the interdiffusion in the interface region have not been characterized so far. The electron-yield XAFS measurements were made on e-beam evaporated $\mathrm{Co} / \mathrm{Pd}$ multilayers with various sublayer thicknesses to investigate the structural variation in the atomic environment for various thickness ratios of Co to Pd sublayers. Also the atomic interdiffusion at the interfaces will be studied in the future with the glancing angle method. ${ }^{4,5)}$

\section{\$2. Experimental}

$\mathrm{Co} / \mathrm{Pd}$ multilayers with a few atomic layers of Co were deposited onto float-glass substrates with a base pressure of $0.5 \sim 1.5 \times 10^{-7} \mathrm{mbar}$ by alternating the electronbeam and thermal evaporation of $\mathrm{Co}$ and $\mathrm{Pd}$ in an ultra high vacuum(UHV) physical vapor deposition(PVD) system(Leybold L 560 UV). The pressure during the de-

- Present addresa: Department of Malerial Science and Engineering. Kunsan National University, Kunsan, 573-360, Korea. position was $0.5 \sim 2.0 \times 10^{-6}$ mbar. A quartz crystal oscillator interfaced with XTC(crystal thickness controller) was used to monitor the layer thickness, and the evaporation rate of $0.1 \sim 0.3 \mathrm{~A} / \mathrm{sec}$ was used. $\mathrm{Co} / \mathrm{Pd}$ multilayers with various sublayer thicknesses such as $3 \AA / 4 \AA$, $3 \dot{A} / 15 \dot{A}$, and $15 \dot{A} / 4 \dot{\AA}$ were prepared in this system and had the total thickness of about 100 or $200 \mathrm{~A}$. Two samples of $3 \AA / 4 \AA$ were prepared with and without a predeposition of a $65 \AA$ thick Pd layer. Also a $230 \AA$-thick Co film and a $120 \AA$ thic: Pd film were prepared. Two other samples were deposited onto quartz substrates by e-beam evaporation of $\mathrm{Co}$ and $\mathrm{Pd}$ in a different vacuum system maintained at about $2 \times 10^{-6}$ Torr during the deposition, and had the total thickness of $1300 \AA$ with the $\mathrm{Co} / \mathrm{Pd}$ sublayer thicknesses of $2.1 \AA / 13.5 \AA$ and $2.2 \AA / 4.5 \AA$. Using a He-flow electron detector, electronyield XAFS measurements were made on these samples and a $7 \mu$ thick Co foil at the beamline X-11A of the National Synchrotron Light Source. The electron beam energy was $2.523 \mathrm{GeV}$ and the current was about 200 $\mathrm{mA} . \mathrm{A} \mathrm{N}_{2}$ gas flow was used for an $\mathrm{I}_{0}$ chamber. $\mathrm{Si}$ (111) monochromator crystals in a two-crystal mode were used and the resolution was about $1 \mathrm{eV}$ at the Co K-edge energy. The energy were calibrated by using a $\mathrm{Cu} \mathrm{K}$ edge near-edge feature and harmonics were rejected by detuning. Figure 1 shows a typical raw data. The samples were characterized with X-ray diffraction(XRD) and some magnetic measurements will be also made on the same samples.

\section{§3. Results and Discussion}

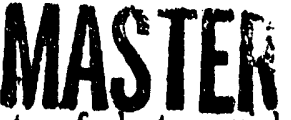

Figure 1 shows a typical raw data of electron-yield XAFS spectrum and a fluorescence corrected spectrum. The data collected in electron detection mode were cor-

ISTHTION OF THIS DOCUMENT IS UNLIMITEA 


\section{DISCLAIMER}

This report was prepared as an account of work sponsored by an agency of the United States Government. Neither the United States Government nor any agency thereof, nor any of their employees, makes any warranty, express or implied, or assumes any legal liability or responsibility for the accuracy, completeness, or usefulness of any information, apparatus, product, or process disclosed, or represents that its use would not infringe privately owned rights. Reference herein to any specific commercial product, process, or service by trade name, trademark, manufacturer, or otherwise does not necessarily constitute or imply its endorsement, recommendation, or favoring by the United States Government or any agency thereof. The views and opinions of authors expressed herein do not necessarily state or reflect those of the United States Government or any agency thereof. 


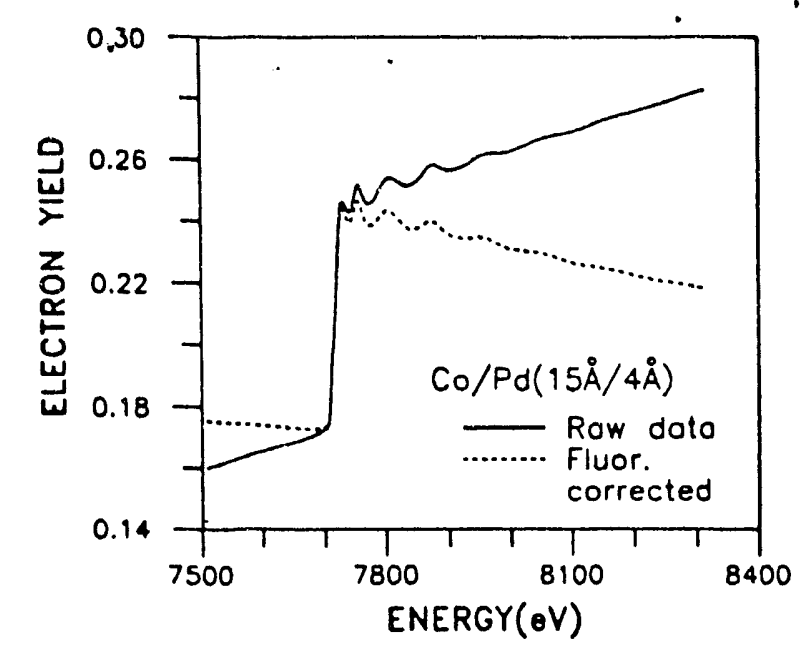

Fig. 1. Comparison of the XAFS spectra before and aster the fluorescence correction.

rected by the fluorescence correction program(FLCORR) which divides out the energy dependence of the $I_{0}$ chamber and thus enables the comparison of the data with transmission data. The Co K XAFS data for $\mathrm{Co} / \mathrm{Pd}$ samples with various thickness ratios of $\mathrm{Co} / \mathrm{Pd}$ were Fourier transformed, and the major peaks for the $3 \dot{\AA} / 15 \AA$ and $3 \AA / 4 \AA$ samples showed a splitting. The Fourier transform peak for the $15 \AA / 4 \AA$ sample was similar to that for pure Co. These Fourier transforms are shown in comparison in Fig. 2. The quality of data for the $3 \AA / 4 \AA$ sample with a predeposition of $\mathrm{Pd}$ was better, and the data for that sample was used in the analysis. The Fourier transform of the Pd K-edge XAFS for the same sample did not show a splitting, and the XRD also showed a single sharp peak. The previous XRD studies did not show a sharp peak for a Pd thin film, showed only a weak bump for a Co-Pd alloy thin film, and showed no peak for a Co thin film.6) In comparison, the major XRD peaks for Co/Pd multilayers were sharp and corresponded to the $\mathrm{d}$ spacings of the (111) plane, and the (111) d spacing for Co/Pd multilayers with different thickness ratios of $\mathrm{Co} / \mathrm{Pd}$ are known to be roughly equal to the weighted average of the (111) d-spacing values of $\mathrm{Co}$ and $\mathrm{Pd}$. Since the multilayer samples are only about 100 \& o 200 \& thich, the small sateilite peali for $n=-1$ near the main peak is hard to detect without a thin film attachment, and only the $15 \AA / 4 \AA$ sample showed a good satellite peak. The XRD results will be presented in another paper after the satellite regions are more carefully scanned with a very high power $\mathrm{X}$-ray diffractometer. Though an XRD peak around $40^{\circ}$ cannot give an accurate $d$-spacing value, the $d$ spacing for a multilayer sample roughly corresponded to the weighted average of Co and $\mathrm{Pd} d$ values. Whereas the split peaks in the Fourier transform of the Co $\mathrm{K} X \mathrm{XFS}$ for the $3 \AA / 4 \AA$ showed a separation of $0.46 \AA$. A similar splitting of the Fourier transform peak was also observed in the case of $\mathrm{Co} / \mathrm{Al}$ multilayers, ${ }^{7)}$ and it was of interest whether the split peaks come from the separation of two subshells or the different backscattering amplitudes of different atomic shells at the same radial distance can result a splitting
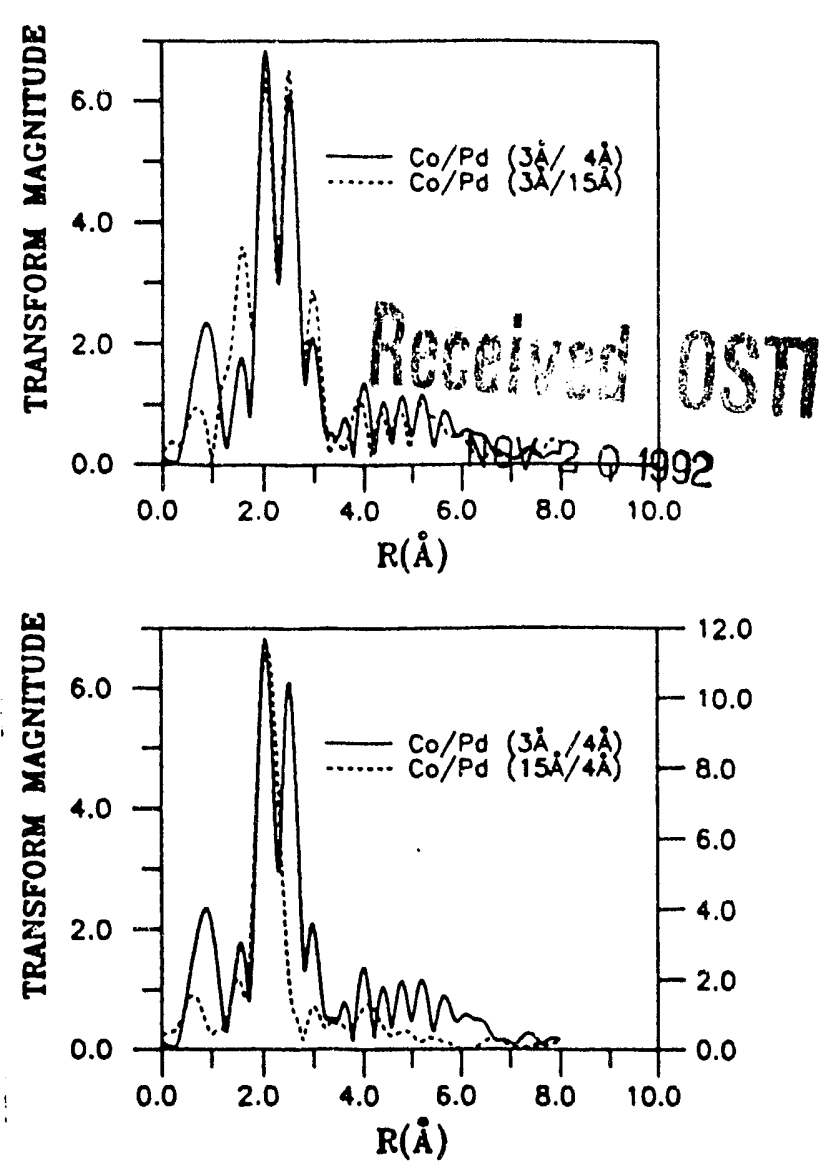

$\mathrm{Fi}_{8}$. 2. Fourier transforms of the Co K-edge XAFS for Co/Pd multilayers with different thickness ratios of Co to $\mathrm{Pd}$ sublayers. (The y-scale on the right side is for the dotted line.)

of the Fourier transform peak due to the interference effect. Thus the Co K XAFS and the Fourier transform for the $3 \AA / 4 \AA$ sample were simulated with the FEFF calculations. In the beginning of the simulation process, first the Co K-edge XAFS data collected in transmission mode for $7 \mu$ foil of pure Co was simulated by using the FEFF parameter values to check the accuracy of the FEFF values. The XAFS of a backscattering Co shell around a Co atom was synthesized using FEFF calculations with $R_{1}=2.5071 \AA$, and the Co $K$ XAFS for $i \mu$ thicl: purs $C o$ foi! was simulated very well with tho parameter values of $N_{1}=12, S_{0}^{2}=0.72, R_{1}=2.49 \AA$, and $\sigma_{1}^{2}=0.0062 \AA^{2}$. The flourescence corrected XAFS for the $230 \AA$ thick Co film was almost identical to the XAFS for the $7 \mu$ thick foil except for a smaller amplitude, and a good fit with FEFF values was obtained for the 230 $\AA$-thick Co film data with $N_{1}=12, S_{0}^{2}=0.73, R_{1}=2.49 \AA$, and $\sigma_{1}^{2}=0.0073 \AA^{2}$. Then the XAFS of a Pd shell around a Co central atom was synthesized using FEFF calculations with $R_{1}=2.641 \AA$ of a CoPd alloy, and the XAFS for the $3 \AA / 4 \AA$ was fitted with a Co standard shell and a $\mathrm{Pd}$ shell thus synthesized by varying the parameters until the experimental XAFS could be simulated well with these two shells. A good fit was obtained with $\mathrm{N}_{1}=8.71, \mathrm{R}_{1}=2.60 \AA, \sigma_{1}^{2}=0.0244 \AA^{2}, \Delta \mathrm{E}_{0}^{1}=-2.3 \mathrm{eV}$, $\mathrm{N}_{2}=3.29, \mathrm{~S}_{0,2}^{2}$ (Pd-shell) $=0.85, \mathrm{R}_{2}=2.60 \AA, \sigma_{2}^{2}=0.0073$ $\AA^{2}$, and $\Delta E_{0}^{2}=-3.5 \mathrm{eV}$. When the Fourier transform 

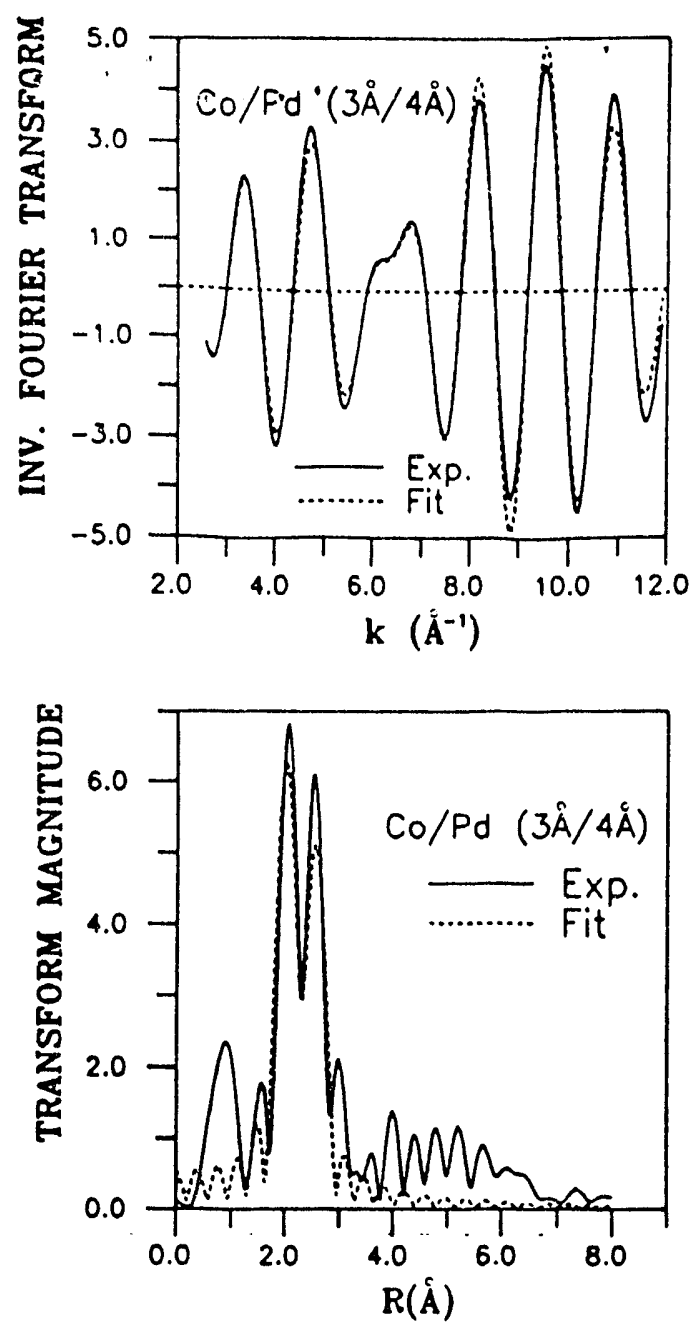

Fig. 3. Comparison of the experimental and simulated $\mathbf{k}^{3}$-weighted $\mathrm{XAFS}$ and Fourier transforms.

of the XAFS for the $3 \AA / 4 \AA$ sample was simulated using these parameters, the simulated Fourier transform showed split peaks with a wider separation even though the two shells were seemingly at the same radial distance. Thus this simulation result showed that the positions of the split peaks cannot be taken for the actually separated positions of two subshells. By using the same $\Delta E_{0}$ values, the $\mathrm{XAFS}$ for the $15 \AA / 4 \AA$ sample was also simulated and a good fit was obtained with $N_{1}=9.70, R_{1}=2.53 \AA$, $\sigma_{1}^{2}=0.0141 \AA^{2}, S_{0,2}^{2}(P d$-shell $)=0.85, N_{2}=2.30, R_{2}=2.56$ $\AA$, and $\sigma_{2}^{2}=0.0076 \AA^{2}$. The Fourier transform for the $15 \AA / 4 \AA$ case was also simulated with these parameters. The results of these simulations for the $3 \AA / 4 \AA$ sample are shown in Fig. 3. These $\mathrm{Co} / \mathrm{Pd}$ multilayers have a preferred orientation, and the (111) planes are parallel to the sample plane. A Co atom in this configuration has 6 in-plane atoms, 3 top layer atoms, and 3 bottom layer atoms as nearest neighbors. In case of a good commensuracy and little interdiffusion, a Co atom in the $3 \AA / 4 \AA$ can have about $8.71 \mathrm{Co}$ atoms and $3.29 \mathrm{Pd}$ atoms as nearest neighbors. However the electron detection was made with the $\mathrm{X}$-ray polarized in the sample plane, and thus the effective signal of 6 in-plane atoms corresponds to that of 9. Also the effective number of top or bottom 3 atoms is only 1.5 . When this polarization effect is taken into account, a $\mathrm{Co}$ atom in the $3 \AA / 4 \AA$ sample would seem to have $9.58 \mathrm{Co}$ atoms and $2.42 \mathrm{Pd}$ atoms as nearestneighbors. But with $N_{1}=9.58$ or with a smaller number of $N_{1}$ around 8 , the unique beating feature around $6.3 \mathrm{~A}^{-1}$ in the inverse Fourier transform of $k^{3}$-weighted $X \mathrm{AFS}$ for the $3 \AA / 4 \AA$ case could not be simulated well. Therefore it can be concluded that a Co atom has less Co atoms and more $\mathrm{Pd}$ atoms as nearest neighbors than predicted for a case of no interdiffusion at the interfaces. There must be some interdiffusion and intermixing of Co and $\mathrm{Pd}$ atoms across the interfaces. Due to a large lattice mismatch, interface atoms would have to adjust to an average lattice spacing but the lattice relaxation may be gradual and for the $15 \AA / 4 \AA$ sample the Co atoms in the central region of the $15 \AA$ thick sublayer may still have a smaller than average lattice spacing. It thus se ms that the simulation results for the $15 \AA / 4 \AA$ case are reasonable and the much smaller(larger) number of $\mathrm{Co}(\mathrm{Pd})$ nearest neighbor atoms than expected for a case of no interdiffusion also indicates a considerable amount of interdiffusion at the interfaces. The Co atoms which are smaller and lighter may experience more severe lattice relaxations and may have a larger structural disorder due to various near-neighbor configurations. However for the $3 \AA / 4 \AA$ case, the $\sigma^{2}$ value for the Co shell is too large, and a possibility of two different $\mathrm{Co}-\mathrm{Co}$ distances for in-plane and out-of-plane atomic pairs will be investigated in the future by polarization dependent XAFS measurements using an electron detector operating at low temperature.

\section{\$4. Conclusion}

Electron yield XAFS spectra were measured for evaporated $\mathrm{Co} / \mathrm{Pd}$ multilayers with various sublayer thicknesses and different thickness ratios of $C_{0}$ to $P d$ sublayers. For the $3 \AA / 4 \AA$ and $15 \AA / 4 \AA$ samples, the $\mathrm{k}^{3}$ weighted XAFS and Fourier transforms were simulated, and from the simulation results, it can be concluded that there must be some interdiffusion and intermixing at the interfaces. The simulation parameters for the $15 \AA / 4 \AA$ sample seem to be reasonable. However the fit for the $3 \AA / 4 \AA$ sample cannot be considered to be final, since the $\sigma^{2}$ value for the Co shell is too large. Whether there are two different $\mathrm{Ca}$-Co distances due to the intermixing or the anisotropy needs to be investigated further.

\section{Acknowledgement}

This research work was carried out at the Beamline X$11 \mathrm{~A}$ of the NSLS and was supported by KOSEF, PLS, and USDOE under contract no. DE-AC02-76CH00016.

\section{References}

1) P. F. Carcis: J. Appl. Phys. 63 (1988) 5066.

2) S.-C. Shin and A. C. Palumbo: J. Appl. Phys. 67 (1990) 317.

3) W. R. Bennet, C. D. England, D. C. Person, and C. M. Falco: J. Appl. Phys. 69 (1991) 4384.

4) S. M. Heald, H. Chen, and J. M. Tranquada: Phys. Rev. B38 (1988) 1016.

5) H. Chen and S. M. Heald: J. Appl. Phys. 66 (1989) 1793.

6) S. K. Kim: Thesis, POSTECH, 1991.

7) J. H. Joo, M. Choi, J. G. Lee, and S. M. Heald, Proceedings of the Srd Synchrotron Radiation User's Workshop, POSTECH (1991) 295. 


$$
\begin{gathered}
\text { DATE } \\
\text { FILMED } \\
\text { Oा/20/93 }
\end{gathered}
$$


